\title{
„Neue Normalität des Essens entwickeln“
}

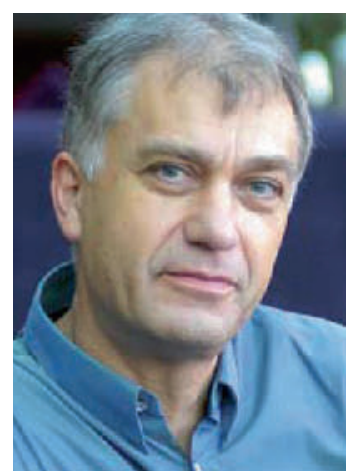

PD Dr. med. Matthias Frank, Neunkirchen

\section{Das Inkretinmimetikum Exenatide (Byetta ${ }^{\circledR}$ ) hilft übergewichtigen Typ-2-Diabetikern abzunehmen. Warum das so ist, und wie der Wirkstoff Patienten im Kampf gegen die Esslust unterstützen kann, erläutert PD Dr. med. Matthias Frank aus Neunkirchen.}

? Wie wirkt Exenatide bei Typ-2-Diabetikern?

Frank: Exenatide verstärkt die Wirkung des Darmhormons GLP-1. So passt es die gestörte Insulinsekretion an den tatsächlichen Bedarf an, bei einer Hypoglykämierate auf Placeboniveau. Die gesteigerte Glukagonsekretion wird normalisiert, die sonst zu einer erhöhten Zuckerfreisetzung aus der Leber führt, und durch eine verzögerte Magenentleerung sowie zentral gesteuerte Mechanismen wird das Sättigungsgefühl verstärkt.

? Wie ist die Wirkung auf den Nüchternblutzucker und die postprandialen Blutzuckerwerte?

Frank: Der postprandiale Effekt ist sehr gut. Die Halbwertszeit der Substanz beträgt etwa acht Stunden, so dass sie täglich zweimal zu den Hauptmahlzeiten injiziert wird. Das muss nicht morgens und abends sein, es kann bei fehlendem Frühstück auch mittags und abends sein. Weil der Nüchtern-BZ durch die Therapie wenig beeinflusst wird, sollte er bei den Patienten erfahrungsgemäß unter $200 \mathrm{mg} / \mathrm{dl}$ liegen. Hier sind die Effekte von Exenatide größer, da durch die „Glukotoxizität“ bei höheren Werten die endogene Glukoseproduktion zusätzlich sinkt.

$$
\text { ? }
$$
Gibt es unerwünschte Nebenwirkungen?

Frank: Der beste verhaltensmedizinisch nutzbare Nebeneffekt ist das beschleunigte Sättigung, das bei weiterer Nahrungszufuhr zu Übelkeit und im Extrem zum Erbrechen führen kann. Der Patient erfährt, dass zu viel und zu schnelles Essen Übelkeit erzeugt. So wird eine neue Normalität des Essens entwickelt, was eine kontinuierliche Gewichtsabnahme ermöglicht. Ein unerwünscher Effekt erzeugt so eine erwünschte Wirkung.
? Wie gestaltet sich der Einsatz des Wirkstoffs? Frank: Viele Menschen sind übergewichtig, weil sie nicht aus Hunger, sondern aus Gewohnheit essen. Man kann lernen, Hunger und Lust zu unterscheiden. Wichtig ist nicht $\mathrm{zu}$ essen, wenn man keinen Hunger hat. Dazu gehört, Hungergefühl wieder wahrzunehmen. Wir sollten die Sättigung bis zur Übelkeit gezielt nutzen. Diese tritt fast nur im Zusammenhang mit dem Essen und meist drei bis vier Monate lang auf. In dieser Zeit kann das Wahrnehmen des Hungergefühls wieder erlernt werden. Ein strukturiertes Trainingsprogramm, das dieses Vorgehen unterstützt, fehlt bisher.

? Wie intensiv betreuen Sie die Patienten? Frank: Die Patienten müssen begleitet werden, weil sie ein anderes Lebensverständnis entwickeln und diese neue Normalität bestätigt werden muss. Wir begleiten sie regelmäßtig über Monate und schließen intern einen Vertrag mit ihnen, der eine Gewichtsabnahme von $2 \mathrm{~kg}$ pro Monat vorsieht. Dabei kann auch ein vorübergehenden Stillstand auftreten. Hierdurch wird aber vermittelt, dass die Patienten mitarbeiten müssen. Es können erhebliche Gewichtsreduktionen erreicht werden, die oft $20 \mathrm{~kg}$ überschreiten. Die Prognose wird so verbessert und Lipidsenker und Antihypertensiva können reduziert werden, was Kosten spart. Unsere Patienten halten die erreichte $\mathrm{HbA}_{1 c^{-}}$und $\mathrm{Ge}$ wichtsverbesserung langfristig. Ein zeitgemäßes Antidiabetikum ermöglicht es also, Stoffwechselnormalisierung und Gewichtsabbau zu kombinieren.

\section{Interview: Helga Brettschneider}

\section{Blutzuckermessen einfach und präzise}

Das neue Blutzuckermesssystem Pura ${ }^{\mathrm{TM}}$ ist einfach und komfortabel zu bedienen und liefert sehr präzise Messergebnisse. Eine Untersuchung des Instituts für Diabetestechnologie in UIm hat ergeben: Die Systemgenauigkeit ist vergleichbar mit dem etablierten System OneTouch ${ }^{\circledR}$ Ultra ${ }^{\circledR}$ und erfüllt die in der ISO-Norm definierten Akzeptanzkriterien. Für die zwei Testreifenchargen des neuen Systems von Ypsomed befanden sich 98,5\% bzw. 100\% der Messungen innerhalb der durch die Norm angegebenen Grenzen. Bei einer Glukosekonzentration unterhalb von 75 mg/dl lagen $97 \%$ bzw. 89\% innerhalb des Spielraums von $5 \mathrm{mg} / \mathrm{dl}$ nach oben und unten. Die Untersuchung erfolgte mit 100 Probanden, als Referenzverfahren diente die Glukoseoxidasemethode. Ein weiterer Pluspunkt des Messsystems: Die breiten, stabilen Teststreifen für das neue Blutzuckermessgerät werden von oben quer in das Gerät mit dem attraktiven weißen Design eingesetzt, woduch Anwender nicht mehr mit Blut in Kontakt kommen. 